\title{
Comparison of proton therapy techniques for treatment of the whole brain as a component of craniospinal radiation
}

\author{
Jeffrey Dinh ${ }^{1}$, Joshua Stoker ${ }^{2}$, Rola H Georges ${ }^{2}$, Narayan Sahoo ${ }^{2}$, X Ronald Zhu ${ }^{2}$ Smruti Rath ${ }^{1}$, Anita Mahajan ${ }^{1}$
} and David R Grosshans ${ }^{* *}$

\begin{abstract}
Background: For treatment of the entire cranium using passive scattering proton therapy (PSPT) compensators are often employed in order to reduce lens and cochlear exposure. We sought to assess the advantages and consequences of utilizing compensators for the treatment of the whole brain as a component of craniospinal radiation (CSI) with PSPT. Moreover, we evaluated the potential benefits of spot scanning beam delivery in comparison to PSPT.
\end{abstract}

Methods: Planning computed tomography scans for 50 consecutive CSI patients were utilized to generate passive scattering proton therapy treatment plans with and without Lucite compensators (PSW and PSWO respectively). A subset of 10 patients was randomly chosen to generate scanning beam treatment plans for comparison. All plans were generated using an Eclipse treatment planning system and were prescribed to a dose of $36 \mathrm{~Gy}(\mathrm{RBE})$, delivered in 20 fractions, to the whole brain PTV. Plans were normalized to ensure equal whole brain target coverage.

Dosimetric data was compiled and statistical analyses performed using a two-tailed Student's t-test with Bonferroni corrections to account for multiple comparisons.

Results: Whole brain target coverage was comparable between all methods. However, cribriform plate coverage was superior in PSWO plans in comparison to PSW (V95\%; $92.9 \pm 14$ vs. $97.4 \pm 5, \mathrm{p}<0.05)$. As predicted, PSWO plans had significantly higher lens exposure in comparison to PSW plans (max lens dose Gy(RBE): left; $24.8 \pm 0.8$ vs. $22.2 \pm$ $0.7, p<0.05$, right; $25.2 \pm 0.8$ vs. $22.8 \pm 0.7, p<0.05$ ). However, PSW plans demonstrated no significant cochlear sparing vs. PSWO (mean cochlea dose Gy(RBE): $36.4 \pm 0.2$ vs. $36.7 \pm 0.1, p=N S$ ). Moreover, dose homogeneity was inferior in PSW plans in comparison to PSWO plans as reflected by significant alterations in both whole brain and brainstem homogeneity index (HI) and inhomogeneity coefficient (IC). In comparison to both PSPT techniques, multi-field optimized intensity modulated (MFO-IMPT) spot scanning treatment plans displayed superior sparing of both lens and cochlea (max lens: $12.5 \pm 0.6$ and $12.9 \pm 0.7$ right and left respectively; mean cochlea $28.6 \pm 0.5$ and $27.4 \pm 0.2)$, although heterogeneity within target volumes was comparable to PSW plans.

Conclusions: For PSPT treatments, the addition of a compensator imparts little clinical advantage. In contrast, the incorporation of spot scanning technology as a component of CSI treatments, offers additional normal tissue sparing which is likely of clinical significance.

Keywords: Protons, CSI, Whole brain, Compensator, Passive scattering proton therapy, Spot scanning, Proton therapy, IMPT

\footnotetext{
* Correspondence: dgrossha@mdanderson.org

${ }^{1}$ Departments of Radiation Oncology, The University of Texas M.D. Anderson Cancer Center, 1515 Holcombe Blvd., Unit 1150, Houston, TX 77030, USA

Full list of author information is available at the end of the article
} waiver (http://creativecommons.org/publicdomain/zero/1.0/) applies to the data made available in this article, unless otherwise stated. 


\section{Introduction}

For treatment of the entire craniospinal axis, many practitioners consider proton therapy the radiation modality of choice [1,2]. The physical advantages of proton therapy for treatment of the spinal target are immediately apparent when comparisons of proton vs. photon spinal fields are made [3]. Additionally, benefits for particle therapy are seen when utilized for treatment of boost fields, such as sparing of the temporal lobes for patients with posterior fossa tumors [4].

The majority of proton treatments have been delivered using PSPT in which brass apertures are utilized to shape the lateral aspects of a large spread out proton beam [5]. The range of the proton beam, or distal edge, is controlled through the use of compensators. Compensators function to adjust the range of the beam across the target in order to conform the distal edge to the geometry of the target volume. For treatment of whole brain fields, as a component of CSI, compensators are commonly utilized in an attempt to reduce dose to cochleae and lenses [6]. However, the introduction of material into the beam path may inadvertently introduce dose heterogeneity, increase range uncertainty and in theory increase neutron contamination [7]. In contrast, with spot scanning proton therapy (SSPT), a pristine pencil beam is magnetically scanned lateral to the beam path and different energies are used to achieve the desired depth distributions [8-11].

In the current study we sought to evaluate the dosimetric consequences of utilizing compensators for PSPT in craniospinal radiation both for organs at risk and dose homogeneity, in a large cohort of brain tumor patients. We also sought to evaluate the potential benefits of spot scanning for such patients.

\section{Methods}

Fifty consecutive brain tumor patients treated with craniospinal radiation were included. All patients were consented for and enrolled on prospective studies of proton therapy approved by the University of Texas MD Anderson Cancer Center institutional review board. Patient demographics and tumor histologies are presented in Table 1. Organs at risk (OARs) including the lens and cochlea along with target volumes (whole brain and cribriform plate) were contoured on the simulation computed tomography scan and each reviewed by a staff radiation oncologist. An Eclipse treatment planning system (Varian Medical Systems, Palo Alto, CA) was used for dose calculations and all plans generated using $2.5 \mathrm{~mm}$ slice spacing. For this retrospective study, for each patient PSPT had been previously planned and delivered using a compensator which was manually edited in order to spare both cochlea and lens OARs as much as possible, while maintaining target coverage. For the present study, clinical PSW plans were copied and PSWO plans retrospectively generated by deletion of
Table 1 Patient characteristics $(n=50)$

\begin{tabular}{|c|c|c|}
\hline Number of patients & & \\
\hline & Male & 30 \\
\hline & Female & 20 \\
\hline \multicolumn{3}{|l|}{ Histology } \\
\hline & Medulloblastoma & 23 \\
\hline & Germ cell tumor & 11 \\
\hline & PNET & 4 \\
\hline & ATRT & 4 \\
\hline & Ependymoma & 2 \\
\hline & Choroid plexus carcinoma & 2 \\
\hline & Glioma & 2 \\
\hline & Ganglioglioma & 1 \\
\hline & Pinealoblastoma & 1 \\
\hline
\end{tabular}

Abbreviations: PNET primitive neuroectodermal tumor; ATRT atypical teratoid rhabdoid tumor.

the compensator and dose-recalculated with the same beam line. In order to facilitate comparison, both PSW and PSWO plans were generated for a prescription dose of $36 \mathrm{~Gy}(\mathrm{RBE})$ in 20 fractions for all patients. For PSPT, the clinical target volume (CTV) was used for planning according to standard of practice, as described previously [12]. Two posterior oblique beams were utilized both for PSW and PSWO plans, as posteriorly angled beams have been shown to contribute to sparing of the lens while allowing adequate coverage of the cribriform plate [13].

A subset of 10 patients was subsequently chosen for planning with multi-field optimized intensity modulated proton therapy (MFO-IMPT) [14]. Because a robust optimization technique [15] is not currently available in our clinical treatment planning system, for IMPT planning, a planning target volume (PTV) was used for optimization, which included both setup and range uncertainties, in line with our current clinical practice. For cochleae, the planning organ at risk volume (PRV) was defined as a 5-mm expansion from the cochleae. The optimization volume was then defined as PTV minus PRV for cochleae. The spot spacing was 7 to $9 \mathrm{~mm}$. The lateral field margin in the beams-eyeview was set equal to $8 \mathrm{~mm}$, i.e., one spot was allowed to be outside the optimization volume [16]. A 1-cm width, doselimiting ring peripheral to optimization volume was used to shape the dose gradient exterior to target, and to eliminate boundary hot spots. Lenses and cochleae were nominally constrained to 10 and $28 \mathrm{~Gy}$, respectively. The optimization included the cribriform plate as an additional target volume to facilitate prescription dose coverage. A $6.7 \mathrm{~cm}$ thick range shifter was placed at the end of the nozzle to enable coverage of shallow target volume regions. The air gap was kept as small as possible to minimize the spot size and yet large enough to have the sufficient clearance for treatment delivery. 
In all cases, treatment planning was performed by dosimetrists and medical physicists experienced with each modality. Qualitative and quantitative evaluations were conducted for each treatment plan generated. Dosimetric data were compiled including mean cochlear dose (left and right), maximum lens dose (left and right), maximum brainstem dose etc. To evaluate target coverage, V95\% was evaluated for the whole brain as well as cribriform plate. To evaluate dose homogeneity we calculated both the homogeneity index ( $\mathrm{HI}=\mathrm{D} 5 / \mathrm{D} 95)$ as well as the inhomogeneity coefficient (IC = D5-D95/Dmean) [17,18]. For each index a lower value indicates superior dose homogeneity. Statistical significance was determined by a two-tailed $t$ test with Bonferroni corrections employed to account for multiple comparisons.

\section{Results}

For the patient cohort investigated, the mean age at simulation was 18 years with a range of 2 to 65 years. Thirty-five patients were $\leq 18$ years of age. Sixty percent of patients were male (Table 1). Forty six percent of patients were treated for medulloblastoma. The second most common indication was germ cell tumor followed by less common histologies (Table 1).

For both PSW and PSWO, whole brain target coverage was comparable (Table 2). However, the V95\% for the cribriform plate was significantly higher for PSWO plans, an anatomical area which, if inadequately covered, may be associated with an increased risk of disease recurrence [19]. We next compared PSW and PSWO treatment plans in terms of OAR exposure. As expected, without the capacity for distal blocking offered by the addition of a compensator, PSWO plans had significantly higher maximum lens doses (Figure 1A). However, the addition of a compensator, offered no significant cochlear sparing (Figure 1B). Furthermore, qualitative review of plans suggested additional dose heterogeneity within the brainstem for PSW (Figure 1C). In order to quantitatively compare plan heterogeneity, we compared the homogeneity index (HI) and inhomogeneity coefficients (IC) for each plan type both for whole brain and brainstem. In comparison to plans generated with a compensator, PSWO plans were significantly more homogenous (Table 3 ). This was true both

Table 2 Comparison of target volume coverage

\begin{tabular}{lcc}
\hline Index & PSW (Gy(RBE) or \%) & PSWO (Gy(RBE) or \%) \\
\hline V95\% - whole brain & $98.5 \pm 2.7$ & $98.5 \pm 2.8$ \\
D95 - whole brain & $36.2 \pm 0.9$ & $36.1 \pm 0.5$ \\
V95\% - cribriform plate & $92.9 \pm 14$ & $97.4 \pm 5^{*}$ \\
\hline
\end{tabular}

Abbreviations: PSW passive scatter with compensator; PSWO passive scatter without compensator; $\mathrm{V} 95 \%=$ percentage of the target volume that receives at least $95 \%$ of the prescribed dose; D95 = dose volume histogram (DVH) curve dose representing $95 \%$ of volume of the target. Data presented as mean \pm standard deviation; *significant vs. PSW $(p<0.05)$, Student's t-test with Bonferroni correction for multiple comparisons. for the whole brain as well as for the brainstem where the magnitude of change was greater. This is presumably due to the close proximity of the brainstem and cochlea, where steep compensator edits would be expected to degrade plan homogeneity (Figure 1C).

Based on the lack of cochlear sparing observed with both PSPT techniques. We next investigated the potential utility of spot scanning. Multi-field optimized IMPT plans, encompassing the cranium and cervical spine, were created utilizing one posterior (PA) and two anterior-oblique (AO) beams (left and right), all sharing a common isocenter. Employing a PA beam reduced thyroid dose and enabled coverage of the spine target inferior to the shoulder without reimaging, thus reducing the required number of isocenters inferiorly along the spine for most patients. For AO beams, the nominal beam angle was 75 degrees off the medial plane. This placement provided a beams eye view of much of the brain target, unencumbered by the doselimiting cochlea. For the majority of plans, AO beams also included a 15-degree superior couch rotation, facilitating dose reduction to the eyes and lenses, while maintaining cribriform plate coverage. AO beams further ensured that target coverage near the dose sensitive lenses was not principally from the distal portion of the PA proton beam.

IMPT plans displayed target coverage comparable to that of PSW plans (whole brain; V95\% $99.8 \pm 0.15$, D95 $36.5 \pm 0.2$ and cribriform plate; V95\% $96.9 \pm 2.4$, D95 $36.7 \pm 0.3$ ). In comparison to both PSW and PSWO techniques, IMPT plans demonstrated superior OAR sparing (Table 4, Figure 2A). However, utilizing the currently available optimization techniques, heterogeneity within the brain target was inferior compared to PSWO plans (whole brain; HI $1.053 \pm 0.003, \mathrm{p}<0.05$, Figure 2B) but similar when the brainstem was evaluated separately (brainstem; HI 1.04 $\pm 0.008, \mathrm{p}=\mathrm{NS}$ ).

\section{Discussion}

Unnecessary radiation exposure to normal tissues, particularly in pediatric patients, is associated with increased risks of long-term adverse effects [20,21]. Lens and cochlear exposure in particular are associated with cataract formation and decreased hearing acuity respectively [22,23]. The current study, conducted in a large number of patients, supports the results of Jin et al. who also found that the addition of a compensator to PSPT increased heterogeneity [6]. This study adds additional information on the sparing, or lack thereof, of OARs as well as exploring potential benefits of IMPT. We found that the addition of compensators to whole brain treatments, as a component of CSI delivered with PSPT, offered modest lens sparing and little cochlear sparing at the expense of added heterogeneity. Moreover, cribriform plate coverage was superior in PSWO plans compared to PSW. Whole brain treatment plans generated using discrete spot scanning IMPT, 


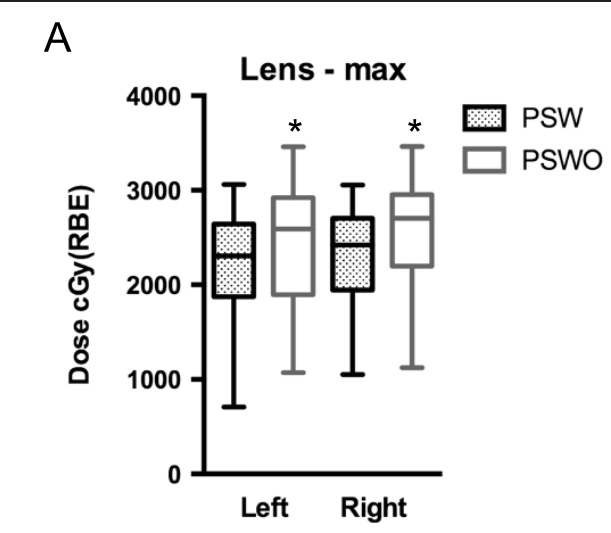

B

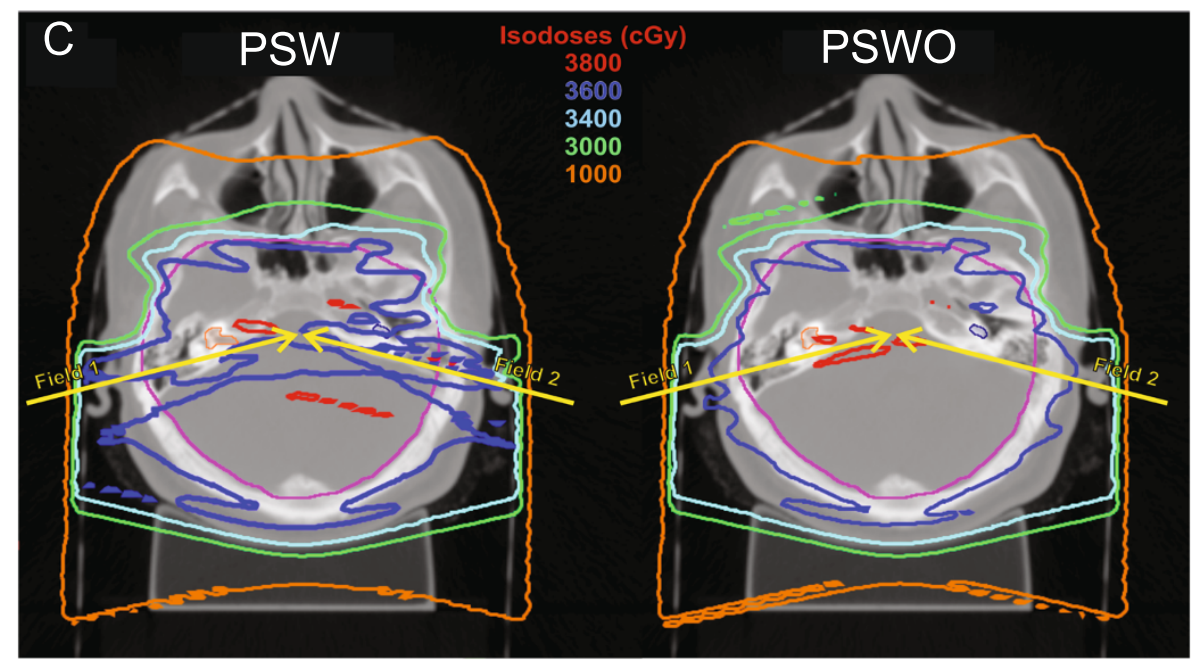

Figure 1 Comparison of PSPT plans with and without compensators. (A) Box-and-whisker plot of maximum lens dose, right and left, for PSW and PSWO plans. Vertical bars represent range and central bar median. (B) Box-and-whisker plot of mean cochlear dose, right and left, for PSW and PSWO plans. (C) Representative axial computed tomographic plans with and without compensators. Axial sections, at the level of the cochlea (highlighted in orange), demonstrate dose heterogeneity introduced by the compensator edge, extending through the brainstem. Yellow arrows depict the beam angles utilized. *significant vs. PSW, $(p<0.05)$, Student's t-test with Bonferroni correction for multiple comparisons.

displayed optimal target coverage along with superior sparing of lens and cochlea in comparison to either PSPT technique. However, dose heterogeneity was increased in IMPT plans.

Sensorineural hearing loss is common following brain irradiation. Especially in pediatric patients, diminished hearing may predispose to impaired communication skills

\section{Table 3 Dose heterogeneity}

\begin{tabular}{lcc}
\hline Index & PSW (Gy(RBE) or \%) & PSWO (Gy(RBE) or \%) \\
\hline HI - whole brain & $1.038 \pm 0.01$ & $1.031 \pm 0.01^{*}$ \\
IC - whole brain & $0.036 \pm 0.01$ & $0.03 \pm 0.01^{*}$ \\
HI - brainstem & $1.064 \pm 0.06$ & $1.028 \pm 0.05^{*}$ \\
IC - brainstem & $0.06 \pm 0.05$ & $0.026 \pm 0.04^{*}$ \\
\hline
\end{tabular}

Abbreviations: PSW passive scatter with compensator; PSWO passive scatter without compensator; $\mathrm{HI}$ (homogeneity index) =D5/D95; IC (inhomogeneity coefficient $=$ D5\% - D95\%/Dmean; Data presented as mean \pm standard deviation. *significant vs. PSW $(p<0.05)$, Student's t-test with Bonferroni correction for multiple comparisons. resulting in diminished cognitive development and ultimately inferior quality of life. For children, treated with radiation alone, it has been suggested that cochlear doses be limited to less than $35 \mathrm{~Gy}$ in order to reduce the risk of ototoxicity [23]. A similar dose response is likely present in adult patients [24]. The addition of platinum based chemotherapy, as in the treatment of medulloblastoma, is expected to further increase the risk of cochlear damage $[25,26]$. In comparison to patients treated with photon techniques, including IMRT, published studies have demonstrated that patients treated to the craniospinal axis with PSPT have favorable hearing outcomes with low rates of high grade hearing loss [27-29]. These results highlight the clinical benefits of proton therapy and are likely due to cochlear sparing during the boost portion of therapy which is superior to photon techniques [2]. However, nearly $50 \%$ of patients did experience low-grade ototoxicity after PSPT based CSI, suggesting further room for improvement [27]. Thus, our finding that IMPT reduced 
Table 4 Organs at risk

\begin{tabular}{lccc}
\hline Index & PSW (Gy(RBE)) & PSWO (Gy(RBE)) & IMPT (Gy(RBE)) \\
\hline Left cochlea (mean) & $36.4 \pm 1.3$ & $36.7 \pm 1.0$ & $28.6 \pm 3.3^{\dagger}$ \\
Right cochlea (mean) & $36.4 \pm 1.4$ & $36.7 \pm 0.9$ & $27.4 \pm 1.5^{\dagger}$ \\
Left lens (max) & $22.2 \pm 5.5$ & $24.8 \pm 6.1^{*}$ & $12.5 \pm 4.0^{\dagger}$ \\
Right lens (max) & $22.8 \pm 5.2$ & $25.2 \pm 5.9^{*}$ & $12.9 \pm 5.0^{\dagger}$ \\
Brainstem (max) & $39.3 \pm 2.0$ & $38.8 \pm 2.0^{*}$ & $38.4 \pm 0.5$ \\
\hline
\end{tabular}

Abbreviations: PSW passive scatter with compensator; PSWO passive scatter without compensator; IMPT intensity modulated proton therapy; Data presented as mean \pm standard deviation; ${ }^{*}$ significant vs. PSW, ${ }^{\dagger}$ significant vs. PSW or PWO, $(p<0.05)$, Student's t-test with Bonferroni correction for multiple comparisons.

cochlear doses compared to PSPT as part of whole brain treatment may have clinical significance.

In contrast to therapy-induced ototoxicity, which is largely irreversible, radiation-induced cataracts may be addressed surgically. However, clinical outcomes following lens replacement may be defined by the health of other remaining ocular structures [30]. Similar to otic structures, the exact dose response of the lens is complicated and influenced by both patient and radiation related factors such as fraction size and dose rate among others [31-33]. Regardless, additional sparing of both lens and other optic structures maybe expected to potentially avoid unnecessary surgical interventions. While we found that lens doses with PSWO plans were significantly higher than PSW plans. IMPT plans demonstrated the best lens sparing, while maintaining cribriform plate coverage. Based on studies of lens sparing during fractionated radiation therapy, the additional sparing offered by IMPT would be expected to reduce the incidence of cataract formation [34].

The current study did not include a comparison of photon based intensity modulated radiation therapy (IMRT). However, previous work has shown that IMRT plans have significantly higher dose heterogeneity in comparison to PSPT plans. However, of note the reported HI values are similar to those we recorded in MFO-IMPT planning [35]. Regardless, given the potential setup uncertainties which would be introduced by patient transfer between photon and proton treatment rooms, mixed modality CSI (IMRT brain and proton spine) is not clinically favored.

In our current clinical practice, we utilize PSPT without the routine use of compensators for treatment of the craniospinal axis. Many new proton centers will have the capacity for spot scanning therapy and some will exclusively employ this modality. The safe delivery of radiation to the entire craniospinal axis is technically challenging regardless of the radiation technique. While published work suggests that CSI delivered with PSPT is safe and efficacious $[1,36]$, additional in silico and clinical studies will be necessary in order to implement CSI treatment using scanned beams. This is highlighted by the present study where scanned beam plans were found to be more inhomogeneous than PSWO plans. Further study including the adaptation of alternate optimizers, novel junctioning techniques etc., is expected to further improve dosimetric outcomes and to make CSI delivered with spot scanning a clinical reality. It is hoped that this will translate into further improvements in outcomes, including reduction of lens and cochlear toxicities.

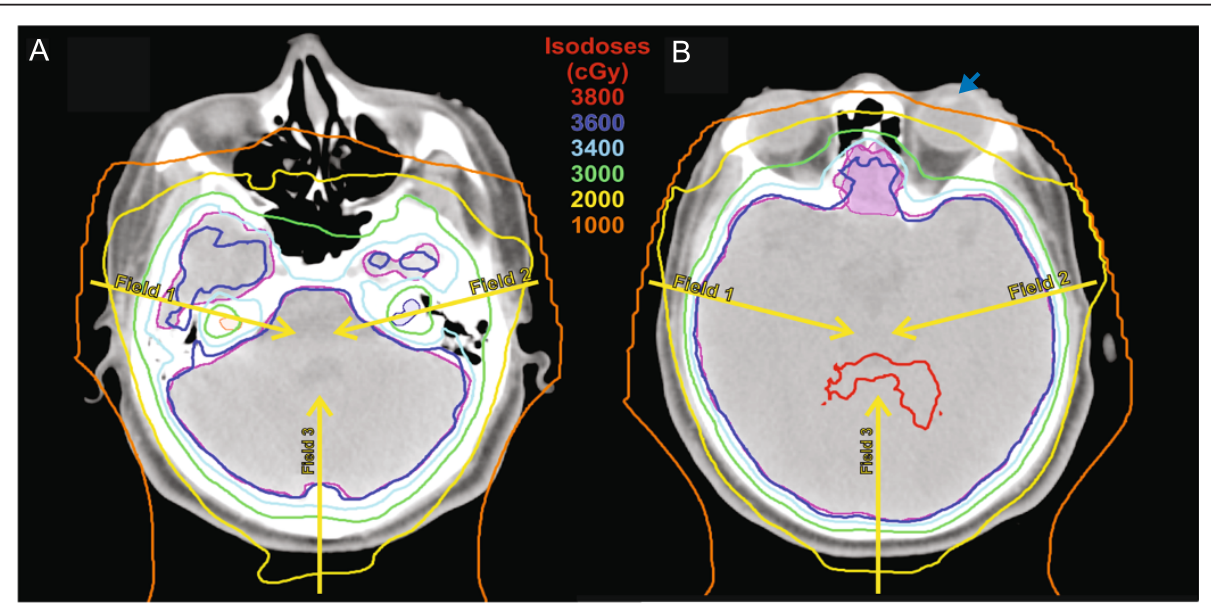

Figure 2 Representative axial sections of a multi-field intensity modulated proton therapy plan. Images demonstrate the capacity for (A) cochlear sparing (depicted in orange and blue color wash, right and left respectively) as well as (B) lens sparing (left lens highlighted by blue arrow) while maintaining coverage of the cribriform plate (opaque magenta). Yellow arrows depict the beam angles utilized. 


\section{Abbreviations}

AO: Anterior-oblique; ATRT: Atypical teratoid rhabdoid tumor; CSI: Craniospinal radiation; CTV: Clinical target volume; DVH: Dose volume histogram; HI: Homogeneity index; IC: Inhomogeneity coefficient; MFO-IMPT: Multi-field optimized intensity modulated; OARs: Organs at risk; PNET: Primitive neuroectodermal tumor; PRV: Planning organ at risk volume; PSPT: Passive scattering proton therapy; PSW: Passive scattering proton therapy with compensator; PSWO: Passive scattering proton therapy without compensator; PTV: Planning target volume; SSPT: Spot scanning proton therapy.

\section{Competing interests}

The authors declare that they have no competing interests.

\section{Authors' contributions}

JD compiled and analyzed dosimetric data and drafted the manuscript. JS developed IMPT methodologies and treatment plans, compiled and analyzed dosimetric data and drafted the manuscript. RHG developed PSPT plans and compiled dosimetric data. NS conceived of the concept of the study and oversaw its completion. XRZ participated in the development of IMPT methodology and treatment planning. SR compiled and analyzed dosimetric data. AM conceived of the concept study and participated in its completion. DRG conceived of the study concept, participated in all aspects of its design and coordination and helped to draft the manuscript. All authors read and approved the final manuscript.

\section{Author details}

${ }^{1}$ Departments of Radiation Oncology, The University of Texas M.D. Anderson Cancer Center, 1515 Holcombe Blvd., Unit 1150, Houston, TX 77030, USA. 2Departments of Radiation Physics, Division of Radiation Oncology, The University of Texas M.D. Anderson Cancer Center, Houston, TX, USA

Received: 9 September 2013 Accepted: 8 November 2013

Published: 17 December 2013

\section{References}

1. Brown AP, Barney CL, Grosshans DR, McAleer MF, de Groot JF, Puduvalli VK, Tucker SL, Crawford CN, Khan M, Khatua S: Proton beam craniospinal irradiation reduces acute toxicity for adults with medulloblastoma. Int J Radiat Oncol Biol Phys 2013, 86:277-284. Doi: 210.1016/j. ijrobp.2013.1001.1014.

2. St Clair WH, Adams JA, Bues M, Fullerton BC, La Shell S, Kooy HM, Loeffler JS, Tarbell NJ: Advantage of protons compared to conventional X-ray or IMRT in the treatment of a pediatric patient with medulloblastoma. Int J Radiat Oncol Biol Phys 2004, 58:727-734.

3. Amsbaugh MJ, Grosshans DR, McAleer MF, Zhu R, Wages C, Crawford CN, Palmer M, De Gracia B, Woo S, Mahajan A: Proton therapy for spinal ependymomas: planning, acute toxicities, and preliminary outcomes. Int J Radiat Oncol Biol Phys 2012, 83:1419-1424. Doi: 1410.1016/j. ijrobp.2011.1410.1034.

4. Lin R, Hug EB, Schaefer RA, Miller DW, Slater JM, Slater JD: Conformal proton radiation therapy of the posterior fossa: a study comparing protons with three-dimensional planned photons in limiting dose to auditory structures. Int J Radiat Oncol Biol Phys 2000, 48:1219-1226.

5. DeLaney TF: Proton therapy in the clinic. Front Radiat Ther Oncol 2011 43:465-485.

6. Jin H, Hsi W, Yeung D, Li Z, Mendenhall NP, Marcus RB Jr: Dosimetric characterization of whole brain radiotherapy of pediatric patients using modulated proton beams. J App/ Clin Med Phys 2011, 12:3308.

7. Hall EJ: Intensity-modulated radiation therapy, protons, and the risk of second cancers. Int J Radiat Oncol Biol Phys 2006, 65:1-7.

8. Delaney TF, Kooy HM: Proton and Charged Particle Radiotherapy. Philadelphia: Wolters Kluwer Lippincott Williams \& Wilkins; 2008

9. Haberer T, Becher W, Schardt D, Kraft G: Magnetic scanning system for heavy ion therapy. Nucl Instrum Methods Phys Res A 1993, 330:296-305.

10. ICRU: Prescribing, Recording, and Reporting Proton-Beam Therapy. Washington DC: International Commission on Radiation Units and Measurements; 2007.

11. Pedroni E, Bacher R, Blattmann H, Bohringer T, Coray A, Lomax A, Lin S, Munkel G, Scheib S, Schneider U, et al: The 200-MeV proton therapy project at the Paul Scherrer Institute: conceptual design and practical realization. Med Phys 1995, 22:37-53.
12. Giebeler A, Newhauser WD, Amos RA, Mahajan A, Homann K, Howell RM: Standardized treatment planning methodology for passively scattered proton craniospinal irradiation. Radiat Oncol 2013, 8:32.

13. Cochran DM, Yock TI, Adams JA, Tarbell NJ: Radiation dose to the lens during craniospinal irradiation-an improvement in proton radiotherapy technique. Int J Radiat Oncol Biol Phys 2008, 70:1336-1342. Epub 2007 Oct 1329.

14. Zhang X, Li Y, Pan X, Xiaoqiang L, Mohan R, Komaki R, Cox JD, Chang JY: Intensity-modulated proton therapy reduces the dose to normal tissue compared with intensity-modulated radiation therapy or passive scattering proton therapy and enables individualized radical radiotherapy for extensive stage IIIB non-small-cell lung cancer: a virtual clinical study. Int J Radiat Oncol Biol Phys 2010, 77:357-366. Doi: 310.1016/j. ijrobp.2009.1004.1028. Epub 2009 Aug 1015.

15. Liu W, Li Y, Li X, Cao W, Zhang X: Influence of robust optimization in intensity-modulated proton therapy with different dose delivery techniques. Med Phys 2012, 39:3089-3101

16. Zhu XR, Sahoo N, Zhang X, Robertson D, Li H, Choi S, Lee AK, Gillin MT: Intensity modulated proton therapy treatment planning using single-field optimization: the impact of monitor unit constraints on plan quality. Med Phys 2010, 37:1210-1219.

17. Kataria T, Sharma K, Subramani V, Karrthick KP, Bisht SS: Homogeneity index: an objective tool for assessment of conformal radiation treatments. J Med Phys 2012, 37:207-213. Doi: 210.4103/0971-6203.103606.

18. Boehling NS, Grosshans DR, Bluett JB, Palmer MT, Song X, Amos RA, Sahoo N, Meyer JJ, Mahajan A, Woo SY: Dosimetric comparison of threedimensional conformal proton radiotherapy, intensity-modulated proton therapy, and intensity-modulated radiotherapy for treatment of pediatric craniopharyngiomas. Int J Radiat Oncol Biol Phys 2011, 27:27.

19. Halperin EC: Impact of radiation technique upon the outcome of treatment for medulloblastoma. Int J Radiat Oncol Biol Phys 1996, 36:233-239.

20. Robison LL, Armstrong GT, Boice JD, Chow EJ, Davies SM, Donaldson SS, Green DM, Hammond S, Meadows AT, Mertens AC: The Childhood Cancer Survivor Study: a National Cancer Institute-supported resource for outcome and intervention research. J Clin Oncol 2009, 27:2308-2318. Epub 2009 Apr 2313.

21. Ellenberg L, Liu Q, Gioia G, Yasui Y, Packer RJ, Mertens A, Donaldson SS, Stovall M, Kadan-Lottick N, Armstrong G, et al: Neurocognitive status in long-term survivors of childhood CNS malignancies: a report from the Childhood Cancer Survivor Study. Neuropsychology 2009, 23:705-717.

22. Bhandare N, Jackson A, Eisbruch A, Pan CC, Flickinger JC, Antonelli P, Mendenhall WM: Radiation therapy and hearing loss. Int J Radiat Oncol Biol Phys 2010, 76:S50-57. Doi: 10.1016/j.jirobp.2009.1004.1096.

23. Hua C, Bass JK, Khan R, Kun LE, Merchant TE: Hearing loss after radiotherapy for pediatric brain tumors: effect of cochlear dose. Int J Radiat Oncol Biol Phys 2008, 72:892-899. Doi: 810.1016/j. ijrobp.2008.1001.1050. Epub 2008 Apr 1018.

24. Honore HB, Bentzen SM, Moller K, Grau C: Sensori-neural hearing loss after radiotherapy for nasopharyngeal carcinoma: individualized risk estimation. Radiother Oncol 2002, 65:9-16.

25. Knight KR, Kraemer DF, Neuwelt EA: Ototoxicity in children receiving platinum chemotherapy: underestimating a commonly occurring toxicity that may influence academic and social development. J Clin Oncol 2005, 23:8588-8596

26. Kolinsky DC, Hayashi SS, Karzon R, Mao J, Hayashi RJ: Late onset hearing loss: a significant complication of cancer survivors treated with Cisplatin containing chemotherapy regimens. J Pediatr Hematol Oncol 2010, 32:119-123. Doi: 110.1097/MPH.1090b1013e3181cb8593.

27. Moeller BJ, Chintagumpala M, Philip JJ, Grosshans DR, MCAleer MF, Woo SY, Gidley PW, Vats TS, Mahajan A: Low early ototoxicity rates for pediatric medulloblastoma patients treated with proton radiotherapy. Radiat Oncol 2011, 6:58

28. Paulino AC, Lobo M, Teh BS, Okcu MF, South M, Butler EB, Su J, Chintagumpala M: Ototoxicity after intensity-modulated radiation therapy and cisplatin-based chemotherapy in children with medulloblastoma. Int J Radiat Oncol Biol Phys 2010, 78:1445-1450. Doi: 1410.1016/j. ijrobp.2009.1409.1031. Epub 2010 Mar 1416.

29. Polkinghorn WR, Dunkel IJ, Souweidane MM, Khakoo Y, Lyden DC, Gilheeney SW, Becher OJ, Budnick AS, Wolden SL: Disease control and ototoxicity using intensity-modulated radiation therapy tumor-bed boost for medulloblastoma. Int J Radiat Oncol Biol Phys 2011, 81:e15-20. Doi: 10.1016/j.jrobp.2010.1011.1081. Epub 2011 Apr 1012. 
30. Osman IM, Abouzeid H, Balmer A, Gaillard MC, Othenin-Girard P, Pica A, Moeckli R, Schorderet DF, Munier FL: Modern cataract surgery for radiation-induced cataracts in retinoblastoma. Br J Ophthalmol 2011, 95:227-230. Doi: 210.1136/bjo.2009.173401. Epub 172010 Jun 173424.

31. Merriam GR Jr, Focht EF, Parsons RW: The relative radiosensitivity of the young and the adult lens. Radiology 1969, 92:1114.

32. Britten MJ, Halnan KE, Meredith WJ: Radiation cataract-new evidence on radiation dosage to the lens. $\mathrm{Br} J$ Radiol 1966, 39:612-617.

33. Deeg HJ, Flournoy N, Sullivan KM, Sheehan K, Buckner CD, Sanders JE, Storb R, Witherspoon RP, Thomas ED: Cataracts after total body irradiation and marrow transplantation: a sparing effect of dose fractionation. Int I Radiat Oncol Biol Phys 1984, 10:957-964.

34. Henk JM, Whitelocke RA, Warrington AP, Bessell EM: Radiation dose to the lens and cataract formation. Int J Radiat Oncol Biol Phys 1993, 25:815-820

35. Howell RM, Giebeler A, Koontz-Raisig W, Mahajan A, Etzel CJ, D'Amelio AM Jr, Homann KL, Newhauser WD: Comparison of therapeutic dosimetric data from passively scattered proton and photon craniospinal irradiations for medulloblastoma. Radiat Oncol 2012, 7:1 16. 10.1186/1748-1717X-1187-1116.

36. Jimenez RB, Sethi R, Depauw N, Pulsifer MB, Adams J, McBride SM, Ebb D, Fullerton BC, Tarbell NJ, Yock TI, Macdonald SM: Proton radiation therapy for pediatric medulloblastoma and supratentorial primitive neuroectodermal tumors: outcomes for very young children treated with upfront chemotherapy. Int J Radiat Oncol Biol Phys 2013, 21:017.

doi:10.1186/1748-717X-8-289

Cite this article as: Dinh et al: Comparison of proton therapy

techniques for treatment of the whole brain as a component of craniospinal radiation. Radiation Oncology 2013 8:289.

\section{Submit your next manuscript to BioMed Central and take full advantage of:}

- Convenient online submission

- Thorough peer review

- No space constraints or color figure charges

- Immediate publication on acceptance

- Inclusion in PubMed, CAS, Scopus and Google Scholar

- Research which is freely available for redistribution 